\title{
A comparison of the Genie and Western blot assays in confirmatory testing for HIV-1 antibody
}

\author{
E. L. CHAN, F. SIDAWAY and G. B. HORSMAN \\ Viral Serology Section, Department of Clinical Microbiology, Laboratory and Diseases Control Services Branch, \\ Saskatchewan Health, Regina, Saskatchewan S4S 5W6, Canada
}

\begin{abstract}
The Genie HIV-1/2 kit (Sanofi Diagnostics Pasteur, Montreal, Quebec), a syntheticpeptide solid-phase enzyme immunoassay, was evaluated as a confirmatory assay for HIV-1 antibodies in comparison with Western blot (BioRad, Hercules, CA, USA) on 50 stored HIV-1 antibody-positive sera and the 137 sera yielding repeated positive results in the conventional EIA screen out of 13405 fresh patient sera from Saskatchewan in 1993. The stored HIV-1-positive sera were uniformly positive in the Genie test. Of the 137 EIA screen-positive sera, 33 were uniformly positive and 64 were uniformly negative in Genie and Western blot; 36 were Genie-negative and indeterminate by Western blot; and four were Genie indeterminate, of which one was negative and three were indeterminate by Western blot. All HIV-1 Western blot-indeterminate and Genie-indeterminate sera were negative in radio-immunoprecipitation assay (RIPA) and Western blot for HIV-1 and HIV-2 antibodies performed by a reference laboratory. Genie gave an accurate definitive result for $97 \%$ of EIA positive sera compared with $71 \%$ for Western blot. There was excellent correlation between Genie, Western blot and RIPA results. However, the Genie assay was faster, less costly and yielded fewer indeterminate results than Western blot in confirmatory testing for HIV-1 antibodies.
\end{abstract}

\section{Introduction}

Enzyme immunoassay (EIA) is the standard screening test for antibodies against human immunodeficiency virus type 1 (HIV-1). Positive results are confirmed in repeat EIA, and then validated in more specific systems, usually in Western blot or less commonly by indirect immunofluorescence assay. These validation tests take $4-24 \mathrm{~h}$. Of the interpretative criteria suggested for HIV-1 Western blot [1], those proposed by the Association of State and Territorial Public Health Laboratory Directors/CDC (ASTPHLD/CDC) give the highest percentage of positive and lowest percentage of indeterminate results. Indeterminate Western blot results may represent early seroconversion or reactivity to non-HIV-1 antigens.

The Genie HIV-1/HIV-2 test (Sanofi Diagnostics Pasteur, Montreal, Quebec) is a rapid, syntheticpeptide, solid-phase EIA for antibodies to HIV-1 and HIV-2 in human serum or plasma. These peptides contain highly conserved immunodominant regions of

Received 23 March 1995; revised version accepted 3 Aug. 1995. the transmembrane glycoproteins of HIV-1 (gp41) and HIV-2 (gp36), known to elicit antibodies in infected patients $[2,3]$. Based on the principle of immunoconcentration [4], the test cartridge contains a removable specimen pre-filter, reaction membrane and absorbent pad. The membrane contains four reaction spots for HIV-1, HIV-2, a 'reactive' (positive) control and a 'non-reactive' (negative) control, respectively. The test takes $c .15 \mathrm{~min}$, and the manufacturer claims a sensitivity and specificity of $100 \%$ in screening both low and high risk populations. In this study, the Genie assay was compared with the Western blot (BioRad, Hercules, CA, USA) as a confirmatory assay for sera showing repeated positive results in EIA screening for HIV-1 antibody.

\section{Materials and methods}

\section{Specimens}

Sera (13405) collected from patients throughout Saskatchewan for HIV antibody testing during 1993 were examined. All sera were stored at $4^{\circ} \mathrm{C}$ and tested within 3 days of receipt; none was heat inactivated. A further 50 sera were also tested; these had been found 
previously to be positive for HIV-1 antibody by Western blot and had been stored at $-70^{\circ} \mathrm{C}$.

\section{Enzyme immunoassay}

Sera were tested initially with Sanofi Diagnostics Pasteur, Gentic System HIV-1/HIV-2 EIA assay according to the manufacturer's instructions. Those yielding positive EIA results were repeat tested in duplicate. Repeatedly EIA-positive sera were tested in the Western blot and Genie assays.

\section{Genie assay}

Specimens were processed according to the manufacturer's instructions. Briefly, patient sera and one positive and negative control were diluted 1 in 20 with the diluent and dropper provided to a total volume of $c$. $1000 \mu \mathrm{l}$ and poured on to the pre-filter. After absorption for $2 \mathrm{~min}$, the pre-filter was removed and the membrane was washed by filling the central well with the wash buffer provided. Three drops $(c .150 \mu \mathrm{l})$ of goat anti-human IgG and IgM antibody conjugated with alkaline phosphatase were added and incubated for $2 \mathrm{~min}$ at room temperature. The membrane was washed twice with the wash buffer and three drops of development reagent (3-indoxyl phosphate) were added. After incubation for $5 \mathrm{~min}$ at room temperature, the well was filled with stop solution to prevent further colour development. Results were read after the stop solution had been completely absorbed. An HIV-1positive result was indicated by development of red colouration only on the 'reactive' and HIV-1 spots. Similarly, an HIV-2 positive result was indicated by the colour only on the 'reactive' and HIV-2 spots. A negative result was indicated by colour only in the 'reactive' spot, and an indeterminate result by development of colour in the 'non-reactive' spot.

\section{Western blotting}

The Western blot HIV-1 antibody assay (BioRad) was performed according to the manufacturer's instructions, and interpreted according to the ASTPHLD/CDC criteria. Sera were considered positive if they yielded at least two of the following bands: p24, gp41 and gp120/160; sera yielding no bands were considered negative, and sera yielding any other band pattern were considered indeterminate. Sera yielding indeterminate Western blot results were tested in HIV-1 radioimmunoprecipitation (RIPA) and HIV-2 Western blot assays by the Division of Diagnostics, Bureau of HIV/ AIDS Laboratories and Research at Ottawa, Ontario.

\section{Results and discussion}

Of the 13405 screening sera examined in the conventional EIA screen, $137(1.2 \%)$ were repeatedly positive. Of these positive sera, $64(47 \%)$ gave negative results in both Genie and Western blot; 33 (24\%) were positive in both Genie and Western blot; and 36 (26.3\%) were Genie-negative and Western blot- indeterminate (Table 1). A further four sera (3\%) yielded indeterminate results in the Genie assay; three were Western blot- indeterminate and one was Western blotnegative. All of the Genie-indeterminate and the Western blot-indeterminate sera were negative in RIPA for HIV-1 and for HIV-2 antibodies in the Western blot assay performed by the reference laboratory. All of the stored HIV-1 known positive sera were Genie-positive in complete concordance with the Western blot result.

Laboratory diagnosis of HIV-1 infection usually involves initial screening for antibody to HIV-1 by enzyme immunoassay, with subsequent confirmation by Western blot or indirect immunofluorescence. The Western blot detects antibody to virus-associated antigens, such as the envelope, polymerase and group-specific proteins. Depending upon the Western blot kit used, confirmation takes 4-24 h. Indeterminate Western blot results occur in up to $32 \%$ of healthy adults [5] and, for some patients, results may remain indeterminate in repeat confirmatory tests on further sera. CDC recommend that patients who yield consistently indeterminate Western blot results for $>6$ months should be considered negative for HIV-1 antibodies if they have no known risk factors or clinical symptoms [1]. The extended waiting period results in much anxiety for these patients.

In this comparative study, the Genie assay proved to be a rapid, cost-effective and accurate confirmatory assay for HIV-1 antibody. The proportion of sera yielding indeterminate results in the Genie assay was $3 \%$, compared to $28 \%$ in the BioRad Western blot; none of these sera proved positive in further confirmatory assays. Costs for confirmation of a single specimen, including all controls, were $c$. $\$ 15 /$ sample for the Genie assay compared with $\$ 100$ for Western blot. Processing time was $15 \mathrm{~min}$ for Genie compared with $4 \mathrm{~h}$ in Western blot. The short assay time, ease of operation and low number of indeterminate results make the Genie assay an attractive alternative to the Western blot for rapid confirmation of presumptive HIV-1 antibody-positive results found in EIA screening tests.

Table 1. Results of confirmatory tests for the 137 sera showing repeated positive results for HIV-1 antibody in conventional EIA

\begin{tabular}{lcc}
\hline HIV-1 antibody results in & & \\
\cline { 1 - 2 } Genie assay & Western blot & Number of sera \\
\hline Negative & Negative & 64 \\
Negative & Indeterminate & $36^{*}$ \\
Indeterminate & Negative & $1^{*}$ \\
Indeterminate & Indeterminate & $3^{*}$ \\
Positive & Positive & 33 \\
\hline
\end{tabular}

*Negative for HIV-1 and HIV-2 antibody in further tests at the reference laboratory. 
We thank Dr E. Rud, L. Malloch and the staff from the Bureau of HIV/AIDS Laboratories and Research, Ottawa, Ontario, for performing the RIPA and HIV-2 Western blot confirmatory tests, and Janette Romanuik for preparation of the manuscript.

\section{References}

1. Anon. Interpretation and use of the Western blot assay for serodiagnosis of human immunodeficiency virus type 1 infections. MMWR 1989; 38 Suppl 7: 1-6.

2. Wang JJG, Steel S, Wisniewolski R, Wang CY. Detection of antibodies to human T-lymphotropic virus type III by using a synthetic peptide of 21 amino acid residues corresponding to a highly antigenic segment of gp4l envelope protein. Proc Natl Acad Sci USA 1986; 83: 6159-6063.

3. Gnann JW, McCormick JB, Mitchell S, Nelson JA, Oldstone MBA. Synthetic peptide immunoassay distinguishes HIV type 1 and HIV type 2 infections. Science 1987; 237: 1346-1349.

4. Valkirs GE, Barton R. Immunoconcentration - a new format for solid-phase immunoassays. Clin Chem 1985; 31: 1427-1431. 5. Midthun K, Garrison L, Clements ML et al. Frequency of indeterminate Western blot tests in healthy adults at low risk for human immunodeficiency virus infection. $J$ Infect Dis 1990; 162: $1379-1382$. 